\title{
Theory of extended x-ray absorption fine structure: Single and multiple scattering formalisms
}

\author{
John J. Boland, Stephen E. Crane, and John D. Baldeschwieler \\ Division of Chemistry and Chemical Engineering, California Institute of Technology, Pasadena, \\ California 91125 \\ (Received 9 November 1981; accepted 17 March 1982)
}

\begin{abstract}
A generalized formalism for the EXAFS effect is presented. The full $T$ operator in the Lippmann-Schwinger equation is expanded and the first term and part of the second term are seen to effectively correspond to single scattering EXAFS processes. The latter term includes secondary scattering by the absorber, and within this formalism it is this term that introduces the central atom phase shift. The expression obtained for the single scattering problem is identical with that found by other investigators. A solution to the general EXAFS problem involving an absorbing atom and two neighboring atoms is also presented. The expression differs from that previously reported [B. K. Teo, J. Am. Chem. Soc. 103, 3990 (1981)]. Multiple scattering is seen to contribute increasingly to the EXAFS as the three atoms approach colinearity. Both the amplitude and frequency of the multiple scattering components are functions of the geometry of the system. The model systems studied indicate that, in many cases, multiple scattering effects must be considered in the analysis of non-nearest-neighbor shells. The theory may readily be extended to systems and scattering paths of arbitrary complexity.
\end{abstract}

\section{INTRODUCTION}

Beginning with Kronig in 1932, a number of shortrange order theories ${ }^{1-11}$ have been proposed to explain the post-edge fine structure-the extended $x$-ray absorption fine structure or EXAFS- of the $\mathrm{x}$-ray absorption edge (see reviews of this subject by Lee et al. ${ }^{12}$ Stern ${ }^{13}$ and Azaroff ${ }^{14}$ ). With the exception of Lee's recent work, ${ }^{10}$ all these studies suffer from difficulties in their treatment of the outgoing photoelectron wave, the scattering potential, or the central atom phase shift. Nevertheless, each study arrives at essentially the same result for the oscillatory component of the $K$-edge $\mathrm{x}$ ray absorption cross section:

$$
\begin{aligned}
\chi(k) \equiv \frac{\mu-\mu_{0}}{\mu_{0}}= & -\sum_{j}\left(\hat{e} \cdot \hat{r}_{j}\right)^{2} \frac{|f(\pi, k)|}{k r_{j}^{2}} \\
& \times \sin \left[2 k r_{j}+2 \delta_{1}(k)+\phi(k)\right],
\end{aligned}
$$

where the symbols have the meanings: $k=[2 m(\hbar \omega$ $\left.\left.-E_{0}\right)\right]^{1 / 2} / \hbar$ is the photoelectron wave number; ( $E_{0}$ is the nominal energy of the edge); $f(\pi, k)=|f(\pi, k)| e^{i \phi(k)}$ is the backscattering amplitude; $\delta_{1}(k)$ is the $l=1$ partial wave phase shift due to the central atom potential; $\mu(k)$ is the observed linear absorption coefficient; $\mu_{0}(k)$ is the hy pothetical absorption coefficient in the absence of scattering atoms.

In the case of nonsingle crystal samples, the geometrical factor $\left(\hat{e} \cdot \hat{r}_{j}\right)^{2}$ where $\hat{e}$ is the polarization vector of the incident radiation, averages to a constant. Additional terms to account for thermal effects (the Debye-Waller factor ${ }^{15}$ ) and losses due to inelastic scattering ${ }^{5}$ may also be appended to Eq. (1.1).

Equation (1.1) embodies, among other assumptions, the single-scattering approximation. That is, the photoelectron ejected upon absorption of the incident $x$-ray photon is assumed to scatter off only one neighboring

\footnotetext{
${ }^{a)}$ Research supported National Science Foundation Grant No. CHE79-18401 and National Institutes of Health Grant No. GM21111-09.
}

atom. This description is recognized as being inadequate for non-nearest neighbor atoms. Lee and Pendry ${ }^{9}$ and Ashley and Doniach ${ }^{8}$ have, however, performed detailed multiple scattering calculations. These authors considered multiple scattering processes with path lengths similar to those of single scattering involving more distant atoms. In such instances multiple scattering introduces an additional EXAFS component near the single scattering frequency. The effect was noted to be of particular importance for multiple scattering involving the first and fourth shells of $\mathrm{Cu}$, which are colinear with the absorbing atom. The scattering amplitude is strongly peaked in the forward direction, so that the presence of the first shell atom causes a significant amplification in the EXAFS at the frequency corresponding to fourth shell distance. More recently, Teo ${ }^{28}$ demonstrated the need to consider multiple scattering effects in EXAFS. The expression assumed for the multiple scattering, however, omits important polarization and geometrical factors (see Sec. V).

Our approach separates the single- and multiplescattering contributions of the general problem, and develops computational methods applicable to both. The general three atom system (an absorbing atom and two neighboring atoms) is discussed in detail. The application of the resulting expression for the EXAFS to the two-model system is discussed in Sec. V.

\section{THE GENERAL FORMALISM}

The $\mathrm{x}$-ray absorption cross section in the one-electron and dipole approximations is given by ${ }^{16}$ :

$$
\sigma_{a}=4 \pi^{2} \alpha \hbar \omega|\langle f|\hat{e} \cdot \mathbf{r}| i\rangle|^{2} N(\omega),
$$

where $\alpha$ is the hyperfine structure constant, $\omega$ is the photon frequency, and $N(\omega)$ is the density of final states of the photoelectron. The initial and final states of the system ( $i$ and $f$ ) are both eigenfunctions of an approximate unperturbed Hamiltonian $H$ :

$$
H=-\frac{\hbar^{2}}{2 m} \nabla_{r}^{2}-\frac{Z e^{2}}{r}+V,
$$






FIG. 1. Schematic representation of the final state potential $V$. About each atomic site (including the central atom $c$ ), there exists a sphere of radius $a$ beyond which no scattering will occur. The atomic potentials are assumed to be spherically symmetric.

where $V$ is the total potential seen by the final-state photoelectron. $V$ is represented as a sum of nonoverlapping, spherically symmetric, finite-range potentials centered around each atomic site in the system, including the absorbing atom $c$ (Fig. 1). The potential between such sites is assumed to be constant, and represents the zero of energy.

In order to calculate the matrix element in Eq. (2.1), it is necessary to find the appropriate eigenfunctions of $H$. At energies corresponding to bound $K$ shell electrons (the only initial state considered here), the potentials of the neighboring atoms may be ignored, and the eigenfunction of the resulting Hamiltonian is the usual hydrogenlike wave function:

$$
\langle r \mid i\rangle=\pi^{-1 / 2}\left(\frac{Z}{a_{0}}\right)^{3 / 2} \exp \left(-Z r / a_{0}\right)
$$

Two factors influence the nature of the final state: the potentials of the neighboring atoms, and that of the central atom. For photoelectrons of sufficiently high energy (approximately three times the plasma frequency and higher ${ }^{6}$ ), the attractive potential of the central atom's nucleus, together with the influence of the other bound electrons (though these are not considered explicitly here), becomes negligible, and the Schrödinger equation reduces to

$$
\left(E-H^{0}\right)|f \pm\rangle=V|f \pm\rangle,
$$

where $H^{0}$ is the free-particle Hamiltonian. This equation may be inverted to give the Lippmann-Schwinger equation ${ }^{17}$

$$
\left|f_{ \pm}\right\rangle=|k\rangle+G_{0}^{ \pm} V|f \pm\rangle=|k\rangle+G_{0}^{ \pm} T^{ \pm}|k\rangle,
$$

where $\langle r \mid k\rangle$ are the normalized eigenfunctions of $H^{0}$. We shall use the minus form of the free-particle Green's and $T$ operators, so that $\langle r \mid k\rangle$ corresponds to the outgoing asymptote of the scattering process described by $\langle r \mid f\rangle$. The description of the EXAFS phenomenon is thus expressed in terms of the state of the photoelectron after the scattering process. Furthermore, this choice of asymptote most clearly illustrates the relationship between EXAFS and the modulations observed in electron yield-type experiments.

The full $T$ operator may now be expanded in terms of the operators $t_{j}$ associated with the individual scattering centers at $\mathbf{r}=\mathbf{r}_{i}{ }^{18}$

$$
T=\sum_{j} t_{j}+\sum_{i \neq j} t_{j} G_{0} t_{i}+\sum_{i \neq j, j \neq k} t_{j} G_{0} t_{i} G_{0} t_{k}+\cdots .
$$

Note that successive scattering by the same potential is not permitted.

Substitution of the first two terms of Eq. (2.6) into Eq. (2.5) yields an expression which may be represented symbolically as shown in Fig. 2. The first diagram in Fig. 2 represents the simplest single scattering case: a photoelectron ejected in the direction $\hat{\gamma}_{j}$, and scattered in some direction $\hat{k}$ by the atomic potential at $\mathbf{r}=\mathbf{r}_{j}$. A similar interpretation applies to the second diagram. The remaining diagrams represent double scattering processes, in which the photoelectron is scattered successively by two atomic potentials.

In particular, the third and fourth diagrams in Fig. 2 represent processes for which the second scattering center is the central atom potential. In such processes, the scattering path length is identical with that of the corresponding single scattering process. Accordingly, such terms must also be considered within our single scattering theory. The term corresponding to secondary scattering by the absorber was first discussed by $\mathrm{Lee}^{10}$ and allows a rigorous treatment of the central atom po-

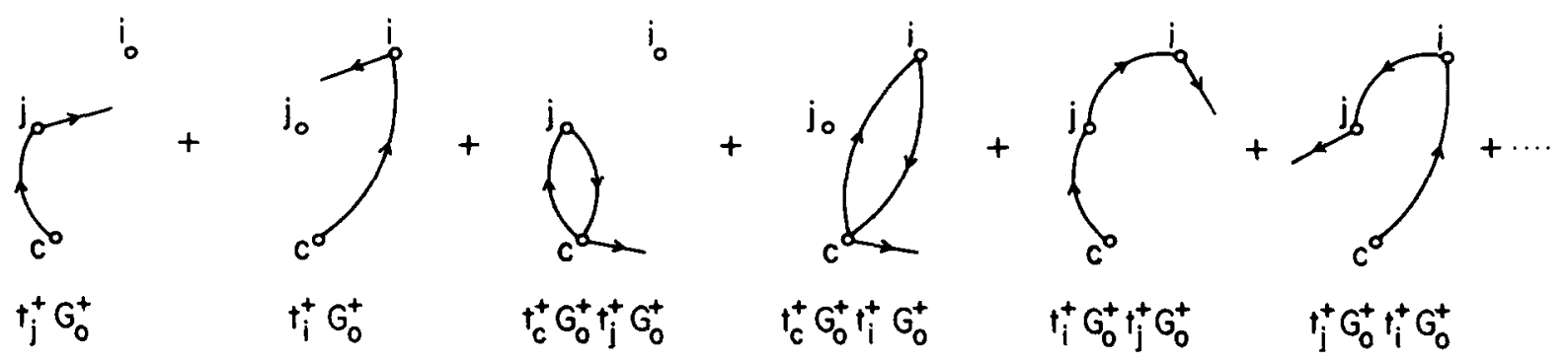

FIG. 2. Diagrammatic representation of the first and second order terms in the expansion of the full $T$ operator Eq. (2.6). The scattering paths shown are those that occur in a system with two neighboring atoms $i$ and $j$. 
tential. The approach adopted, however, was not sufficiently general to be readily applicable in multiple scattering problems.

\section{THE SINGLE SCATTERING FORMALISM}

The two single scattering terms of Eq. (2.6) may now be substituted into the matrix element in Eq. (2.1):

$$
\begin{aligned}
\langle f-|\hat{e} \cdot \mathbf{r}| i\rangle= & \langle k|\hat{e} \cdot \mathbf{r}| i\rangle+\sum_{j}\left\langle k\left|t_{j}^{+} G_{0}^{+} \hat{e} \cdot \mathbf{r}\right| i\right\rangle \\
& +\sum_{j}\left\langle k\left|t_{c}^{+} G_{0}^{+} t_{j}^{+} G_{0}^{+} \hat{e} \cdot \mathbf{r}\right| i\right\rangle,
\end{aligned}
$$

where we have taken the complex conjugate of Eq. $(2,5)$ and have noted that $t\left(z^{*}\right)=[t(z)]^{\dagger}$.

The first matrix element on the right-hand side of Eq. (3.1) is responsible for the usual unperturbed photoelectric effect (i.e., for $\mu_{0}$ ), and is evaluated in Appendix $A$ with the result:

$$
\begin{aligned}
& \langle k|\hat{e} \cdot \mathbf{r}| i\rangle=M(k, Z) \hat{k} \cdot \hat{e}, \\
& M(k, Z)=-\frac{(2)^{1 / 2} i}{\pi} \frac{8 k\left(\frac{Z}{a_{0}}\right)^{5 / 2}}{\left(\frac{Z^{2}}{a_{0}}+k^{2}\right)^{3}} .
\end{aligned}
$$

The remaining terms in Eq. (3.1) may be expanded in complete state to obtain

$$
\begin{aligned}
& \sum_{j}\left\langle k\left|t_{j}^{+} G_{0}^{+} \hat{e} \cdot \mathbf{r}\right| i\right\rangle \\
& \quad=\sum_{j} \int\left\langle k\left|t_{j}^{+}\right| r_{1}\right\rangle\left\langle r_{1}\left|G_{0}^{+}\right| r\right\rangle \hat{e} \cdot \mathbf{r}\langle r \mid i\rangle d \mathbf{r} d \mathbf{r}_{1}
\end{aligned}
$$

and

$$
\begin{gathered}
\sum_{j}\left\langle k\left|t_{c}^{*} G_{0}^{+} t_{j}^{+} G_{0}^{+} \hat{e} \cdot \mathbf{r}\right| i\right\rangle=\sum_{j} \int\left\langle k\left|t_{c}^{+}\right| r_{3}\right\rangle\left\langle r_{3}\left|G_{0}^{+}\right| r_{2}\right\rangle \\
\quad \times\left\langle r_{2}\left|t_{j}^{+}\right| r_{1}\right\rangle\left\langle r_{1}\left|G_{0}^{+}\right| r\right\rangle \hat{\boldsymbol{e}} \cdot \mathbf{r}\langle\boldsymbol{r} \mid i\rangle d \mathbf{r} d \mathbf{r}_{1} d \mathbf{r}_{2} d \mathbf{r}_{3} .
\end{gathered}
$$

The EXAFS effect may be viewed as arising from a difference in phase at the origin between the unperturbed photoelectron and one that has scattered off of a neighboring atom. The simplest description of such a phenomenon is one in which all matrix elements are expressed in terms of their effective values at the origin, and our coordinate system was chosen accordingly (see Fig. 3).

It remains to evaluate matrix elements of the forms:

$$
\left\langle\boldsymbol{r}^{\prime}\left|G_{0}^{+}\right| \boldsymbol{r}\right\rangle \text { and }\left\langle\boldsymbol{r}^{\prime}\left|t_{j}^{*}\right| \boldsymbol{r}\right\rangle \text {. }
$$

The configuration-space matrix element of the Green's operator is given by the corresponding free-particle Green's function:

$$
\left\langle\boldsymbol{r}^{\prime}\left|G_{0}^{+}\right| \boldsymbol{r}\right\rangle=-\frac{m}{2 \pi \hbar^{2}} \frac{e^{i k\left|\mathbf{r}^{\prime}-\mathbf{r}\right|}}{\left|\mathbf{r}^{\prime}-\mathbf{r}\right|} .
$$

Note that $|\boldsymbol{r}|$ is of order $a_{0} / Z$ or smaller while $\mathbf{r}^{\prime}$ is restricted to a domain of radius $a_{j}$ about $\mathbf{r}_{j}$ (Fig. 3). Hence $r^{\prime} \approx r_{j}$ and $\left|r^{\prime}-r\right|$ may be expanded as ${ }^{19}$

$$
\left|\mathbf{r}^{\prime}-\mathbf{r}\right|=r_{f}+\hat{r}_{f} \cdot\left(\mathbf{r}^{\prime}-\boldsymbol{r}_{f}-\mathbf{r}\right)+O\left(a_{j} / r_{j}\right)
$$

and the Green's function in Eq. (3.5) may be approximated:

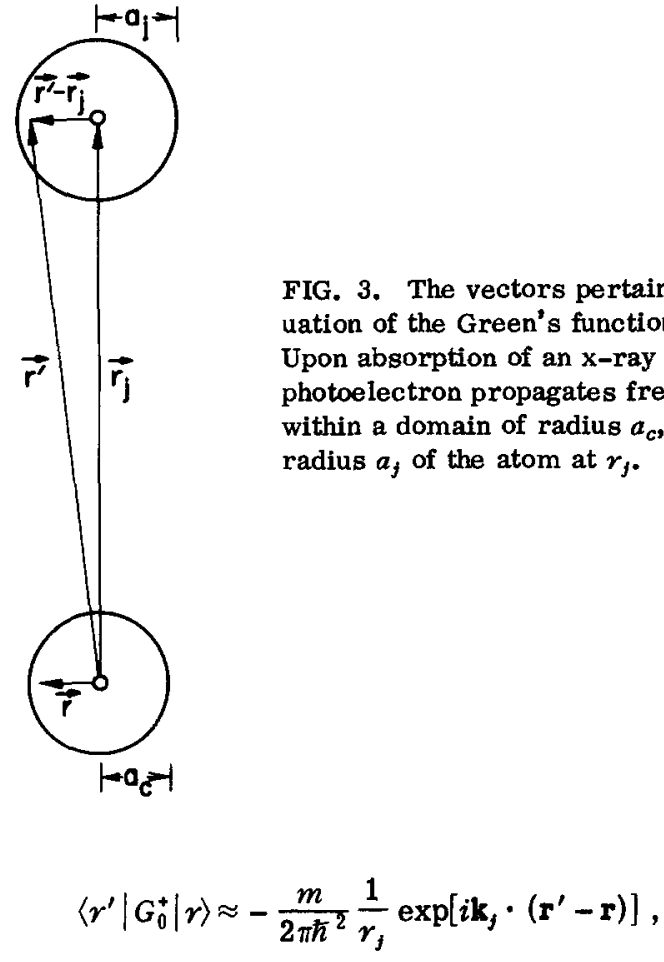

where $\mathbf{k}_{j}=k \hat{r}_{j}$ is the direction of propagation of the photoelectron. The error term $a_{j} / r_{j}$ is small because the core electrons of the neighboring atoms are responsible for most of the scattering at the energies of interest in EXAFS problems. ${ }^{12}$

Substituting the approximate Green's function Eq. (3.7) into the matrix elements Eqs. (3.3) and (3.4) permits us to perform the space integrals in the manner described in Appendix A, with the results:

$$
\begin{aligned}
& \sum_{j}\left\langle k\left|t_{j}^{+} G_{0}^{+} \hat{e} \cdot \mathbf{r}\right| i\right\rangle=-\sum_{j} \frac{m(2 \pi)^{2}}{\hbar^{2}} \frac{1}{r_{j}} M(k, Z)\left(\hat{e} \cdot \hat{r}_{j}\right)\left\langle k\left|t_{j}^{*}\right| k_{j}\right\rangle, \\
& \sum_{j}\left\langle k\left|t_{c}^{+} G_{0}^{+} t_{j}^{+} G_{0}^{+} \hat{e} \cdot \mathbf{r}\right| i\right\rangle=\sum_{j} \frac{m^{2}(2 \pi)^{4}}{\hbar^{4}} \frac{1}{r_{j}^{2}} M(k, Z)\left(\hat{e} \cdot \hat{r}_{j}\right) \\
& \times\left\langle k\left|t_{c}^{*}\right| k_{j}^{\prime}\right\rangle\left\langle k_{j}^{\prime}\left|t_{j}^{+}\right| k_{j}\right\rangle \text {, }
\end{aligned}
$$

where $\mathbf{k}_{j}^{\prime}=-k \hat{r}_{j}$ is the direction of propagation of the backscattered photoelectron.

We may now relate the matrix elements of $t_{j}^{+}\left(r_{j}\right)$ to those of $t_{f}^{0}(0)$ which represent the identical scattering problem, but centered at the origin ${ }^{20}$

$$
\left\langle k\left|t_{j}^{*}\right| k_{j}\right\rangle=\exp \left[i\left(\mathbf{k}_{j}-\mathbf{k}\right) \cdot \mathbf{r}_{j}\right]\left\langle k\left|t_{j}^{0}\right| k_{j}\right\rangle \text {. }
$$

Only elastic scattering events (i.e., $|\mathbf{k}|=\left|\mathbf{k}_{j}\right|$ ) are of interest, so that the matrix elements of $t_{j}^{0}(0)$ form an on-shell $T$ matrix, which may be expressed in terms of the scattering amplitude $f_{j}\left(\theta_{j}\right)^{21}$ :

$$
\left\langle k\left|t_{j}^{0}\right| k_{j}\right\rangle \equiv \frac{-\hbar^{2}}{m(2 \pi)^{2}} f_{j}\left(\theta_{j}\right),
$$

where $\cos \theta_{j}=\hat{k} \cdot \hat{k}_{j}=\hat{k} \cdot \hat{r}_{j}$.

Equations (3.8) and (3.9) may now be rewritten as:

$$
\begin{aligned}
(3.8)= & \sum_{j} M(k, Z) \frac{1}{r_{j}}\left(\hat{e} \cdot \hat{r}_{j}\right) f_{j}\left(\theta_{j}\right) \\
& \times \exp \left[i k r_{f}\left(1-\cos \theta_{j}\right)\right],
\end{aligned}
$$




$$
\begin{aligned}
(3.9)= & \sum_{j} M(k, Z) \frac{1}{r_{j}^{2}}\left(\hat{e} \cdot \hat{r}_{j}\right) f_{j}(\pi) \\
& \times f_{c}\left(\pi-\theta_{j}\right) \exp \left(2 i k r_{j}\right),
\end{aligned}
$$

where $\cos \left(\pi-\theta_{j}\right)=\hat{k} \cdot \hat{k}_{j}^{\prime}=-\hat{k} \cdot \hat{r}_{j}$.

The complete matrix element in Eq. (3.1) is the sum of three terms corresponding to the unperturbed photoelectric effect Eq. (3.2); simple scattering by the atom at $\mathbf{r}_{1}$ Eq. (3.12); and secondary scattering by the absorber Eq. (3.13):

$$
\begin{aligned}
& \sigma_{a}(k) \propto|\langle f-|\hat{e} \cdot \mathbf{r}| i\rangle|^{2} \\
& \quad=|M(k, Z) \hat{k} \cdot \hat{e}+(3.12)+(3.13)|^{2} .
\end{aligned}
$$

The development above treats the absorption of a single $x$-ray photon by an absorber-scatterer system. In an EXAFS experiment, however, a large number of such absorptions will occur, and the ejected photoelectrons will be scattered into many directions $\hat{k}$. In order to compute the average absorption cross section of such a macroscopic system, it is necessary to average over all such directions $\hat{k}$ in Eq. (3.14):

$$
\begin{aligned}
& \int|\langle f-|\hat{e} \cdot \mathbf{r}| i\rangle|^{2} \frac{d \Omega_{k}}{4 \pi} \\
& \quad=\int|M(k, Z)(\hat{k} \cdot \hat{e})+(3.12)+(3.13)|^{2} \frac{d \Omega_{B}}{4 \pi} .
\end{aligned}
$$

The four lowest order terms in $r_{j}$ in this spherical average are evaluated in Appendix B with the results:

$$
\begin{aligned}
& \int|M(k, Z)|^{2}(\hat{k} \cdot \hat{e})^{2} \frac{d \Omega_{b}}{4 \pi}=\frac{1}{3}|M(k, Z)|^{2}, \\
& \int 2 \operatorname{Re}\left[M^{*}(\hat{k} \cdot \hat{e}) \times(3.12)\right] \frac{d \Omega_{k}}{4 \pi} \\
& =-|M|^{2} \sum_{j} \frac{(\hat{e} \cdot \hat{r})^{2}}{k r_{j}^{2}} \operatorname{Im}\left[\exp \left(2 i k r_{j}\right) f_{j}(\pi)+f_{j}(0)\right], \\
& \int|(3.12)|^{2} \frac{d \Omega_{k}}{4 \pi}=|M|^{2} \sum_{j} \frac{\left(\hat{e} \cdot \hat{r}_{j}\right)^{2}}{r_{j}^{2}} \int\left|f_{j}\left(\theta_{j}\right)\right|^{2} \frac{d \Omega_{k}}{4 \pi}, \\
& \int 2 \operatorname{Re}\left[M^{*}(\hat{k} \cdot \hat{e}) \times(3.13)\right] \frac{d \Omega_{k}}{4 \pi}=-|M|^{2} \sum_{j} \frac{\left(\hat{e} \cdot \hat{r}_{j}\right)^{2}}{k r_{j}^{2}} \\
& \quad \times \operatorname{Im}\left\{\left[\exp \left(2 i \delta_{1}\right)-1\right] f_{j}(\pi) \exp \left(2 i k r_{j}\right)\right\} .
\end{aligned}
$$

Note that in summing the above expressions, the forward-scattering term $f_{j}(0)$ in Eq. (3.17) cancels with Eq. (3.18) by virtue of the optical theorem ${ }^{22}$

$$
\operatorname{Im}[f(0)]=\frac{k}{4 \pi} \int|f(\theta)|^{2} d \Omega_{k} .
$$

The macroscopic absorption coefficient $\mu=n \sigma_{a}$ is proportional to

$$
\begin{aligned}
\mu= & n \sigma_{a} \propto \frac{1}{3}|M|^{2}-\sum_{j}|M|^{2}\left(\hat{e} \cdot \hat{r}_{j}\right)^{2} \frac{1}{k r_{j}^{2}} \\
& \times \operatorname{Im}\left\{f_{j}(\pi) \exp \left[2 i\left(k r_{j}+\delta_{j}\right)\right]\right\},
\end{aligned}
$$

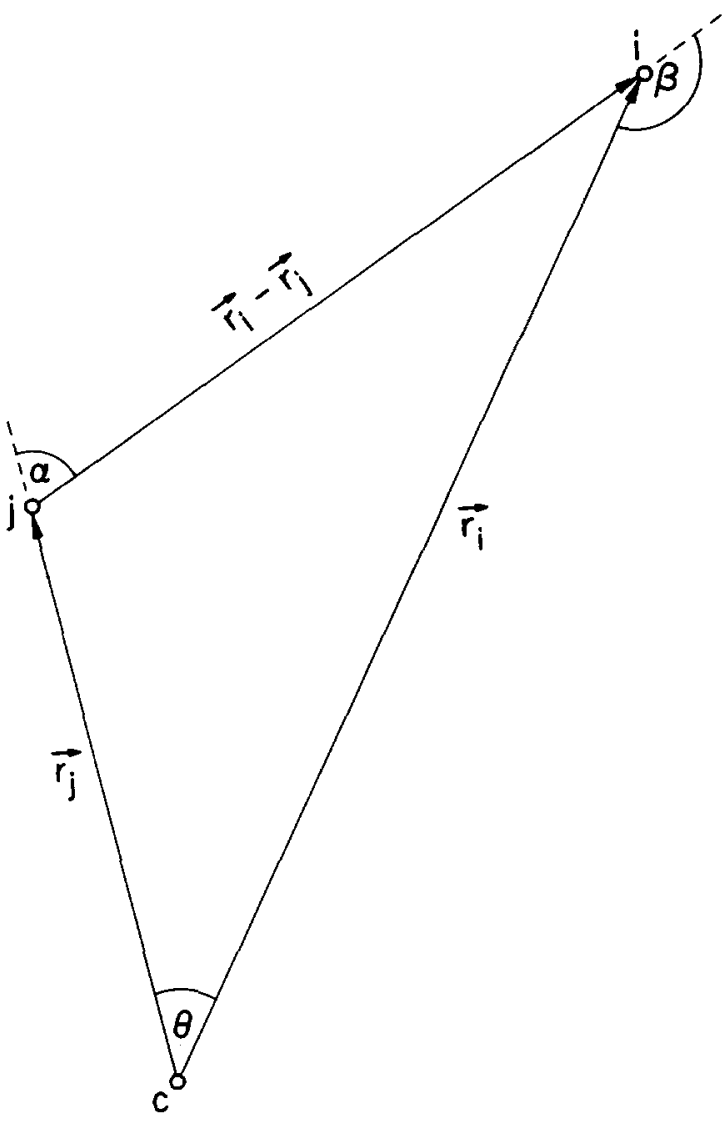

FIG. 4. The three-atom system. $c$ is the central (absorbing) atom and $i$ and $j$ are neighboring atoms, with $\left|r_{j}\right| \leq\left|\mathbf{r}_{i}\right|$. $\pi-\alpha$ is the bridging angle.

where $n$ is the number density of absorbing atoms. By convention, ${ }^{23}$ the oscillatory part of the absorption coef ficient is normalized to $\mu_{0} \propto \frac{1}{3}|M(k, Z)|^{2}$ :

$$
\begin{aligned}
\chi(k) \equiv \frac{\mu-\mu_{0}}{\mu_{0}}= & -\frac{3}{k r_{j}^{2}}\left(\hat{e} \cdot \hat{r}_{j}\right)^{2}\left|f_{j}(\pi, k)\right| \\
& \times \sin \left[2 k r_{j}+2 \delta_{1}(k)+\phi(k)\right],
\end{aligned}
$$

where $f_{j}(\pi, k)=\left|f_{j}(\pi, k)\right| e^{i \phi(k)}$.

\section{THE THREE-ATOM PROBLEM}

The three-atom system to be considered is shown in Fig. 4. Various scattering paths amongst these atoms are represented in Fig. 5. Each path corresponds to a term in the expansion of the full $T$ operator in the Lippmann-Schwinger equation:

$$
T=\sum_{i} t_{i}+\sum_{i \neq j} t_{i} G_{0} t_{j}+\sum_{i \neq j, j \neq k} t_{i} G_{0} t_{j} G_{0} t_{k}+\cdots .
$$

Low probability-amplitude processes involving long path lengths and/or large scattering angles have been omitted from Fig. 5, but may be treated in a manner analogous to that outlined below.

The complete matrix element for the three-atom system in the dipole approximation may be written:

$$
\begin{aligned}
& \langle f-|\hat{e} \cdot \mathbf{r}| i\rangle=\langle k|\hat{e} \cdot \mathbf{r}| i\rangle+\sum_{n=i, j}\left\langle k\left|t_{n}^{+} G_{0}^{+} \hat{e} \cdot \mathbf{r}\right| i\right\rangle+\sum_{n=i, j}\left\langle k\left|t_{c}^{*} G_{0}^{+} t_{n}^{*} G_{0}^{*} \hat{e} \cdot \mathbf{r}\right| i\right\rangle \\
& +\left\langle k\left|t_{j}^{*} G_{0}^{+} t_{i}^{+} G_{0}^{*} \hat{e} \cdot \mathbf{r}\right| i\right\rangle+\left\langle k\left|t_{i}^{*} G_{0}^{+} t_{j}^{+} G_{0}^{+} \hat{e} \cdot \mathbf{r}\right| i\right\rangle+\left\langle k\left|t_{c}^{*} G_{0}^{+} t_{j}^{+} G_{0}^{*} t_{i}^{+} G_{0}^{+} \hat{e} \cdot \mathbf{r}\right| i\right\rangle \\
& +\left\langle k\left|t_{c}^{*} G_{0}^{+} t_{i}^{+} G_{0}^{*} t_{j}^{*} G_{0}^{+} \hat{e} \cdot \mathbf{r}\right| i\right\rangle+\left\langle k\left|t_{j}^{*} G_{0}^{+} t_{i}^{+} G_{0}^{+} t_{j}^{+} G_{0}^{+} \hat{e} \cdot \mathbf{r}\right| i\right\rangle+\left\langle k\left|t_{c}^{*} G_{0}^{+} t_{j}^{*} G_{0}^{*} t_{i}^{*} G_{0}^{*} t_{j}^{*} G_{0}^{+} \hat{e} \cdot \mathbf{r}\right| i\right\rangle .
\end{aligned}
$$




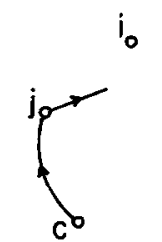

(a) $t_{j}^{+} G_{0}^{+}$

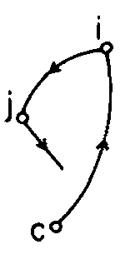

(f) $t_{j}^{+} G_{0}^{+} f_{i}^{+} G_{0}^{+}$



(b) $t_{i}^{+} G_{0}^{+}$



(g) $t_{c}^{+} G_{0}^{+} t_{i}^{+} G_{0}^{+} t_{j}^{+} G_{0}^{+}$

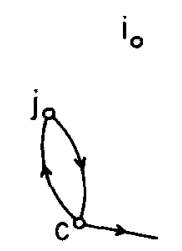

(c) $\mathrm{t}_{\mathrm{c}}^{+} \mathrm{G}_{0}^{+} \mathrm{t}_{\mathrm{j}}^{+} \mathrm{G}_{\mathrm{o}}^{+}$

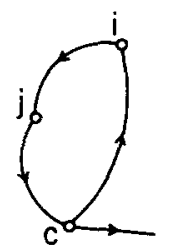

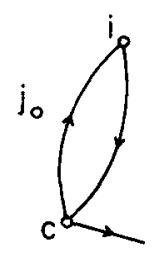

(d) $t_{c}^{+} G_{o}^{+} t_{i}^{+} G_{0}^{+}$



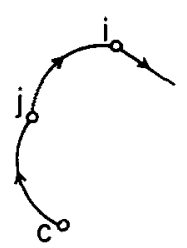

(e) $t_{i}^{+} G_{0}^{+} t_{j}^{+} G_{0}^{+}$

FIG. 5. Scattering paths within the three-atom system. The operators shown with each diagram is the corresponding term in the expansion of the full $T$ operator in the Lippmann-Schwinger equation [Eq. (4.1)].

The first term in Eq. (4.2) corresponds to the unperturbed photoelectric effect, and the subsequent two terms correspond to the single-scattering contributions from atoms $i$ and $j$. These terms have been treated in detail in Sec. III, and will not be discussed further here. The remaining terms in Eq. (4.2) involve scattering by both neighboring atoms. Of these, the fourth and fifth terms [those corresponding to diagrams (e) and (f) in Fig. 5] are identical by virtue of the reciprocity theorem, as are those corresponding to diagrams (g) and (h). The multiple-scattering terms in Eq. (4.2) may thus be written as

$$
2\left\langle k\left|t_{i}^{+} G_{0}^{+} t_{j}^{+} G_{0}^{+} \hat{e} \cdot \mathbf{r}\right| i\right\rangle+2\left\langle k\left|t_{c}^{+} G_{0}^{+} t_{i}^{+} G_{0}^{+} t_{j}^{+} G_{0}^{+} \hat{e} \cdot \mathbf{r}\right| i\right\rangle+\left\langle k\left|t_{j}^{+} G_{0}^{+} t_{i}^{+} G_{0}^{+} t_{j}^{+} G_{0}^{+} \hat{e} \cdot \mathbf{r}\right| i\right\rangle+\left\langle k\left|t_{c}^{+} G_{0}^{+} t_{j}^{+} G_{0}^{+} t_{i}^{+} G_{0}^{+} t_{j}^{+} G_{0}^{+} \hat{e} \cdot \mathbf{r}\right| i\right\rangle .
$$

Each term of Eq. (4.3) may be expanded in complete states and the resulting Green's functions evaluated as described in Sec. III, where

$$
\left\langle r^{\prime}\left|G_{0}^{+}\right| r\right\rangle=-\frac{m}{2 \pi \hbar^{2}} \frac{1}{r_{j}} \exp \left[i \mathbf{k}_{j} \circ\left(\mathbf{r}^{\prime}-\mathbf{r}\right)\right] .
$$

$\mathbf{r}$ is localized about the origin, $\mathbf{r}^{\prime}$ is restricted to a radius $a_{j}$ about $\mathbf{r}_{j}$, and $\mathbf{k}_{j}=k \hat{\mathbf{r}}_{j}$. Green's functions representing free propagation between two neighboring atom potentials may be evaluated by placing one or the other of these atoms at the origin for the purposes of the calculation.

Equation (4.3) may now be written as:

$$
\begin{aligned}
& 2 \frac{M(k, Z)\left(\hat{e} \cdot \hat{r}_{j}\right)}{r_{j} r_{i j}} \frac{m^{2}(2 \pi)^{4}}{\hbar^{4}}\left\langle k\left|t_{i}^{+}\right| k_{i j}\right\rangle\left\langle k_{i j}\left|t_{j}^{+}\right| k_{j}\right\rangle+2 \frac{M(k, Z)\left(\hat{e} \cdot \hat{r}_{j}\right)}{r_{i} r_{j} r_{i j}} \frac{m^{3}(2 \pi)^{6}}{\hbar^{6}}\left\langle k\left|t_{c}^{+}\right| k_{i}^{\prime}\right\rangle\left\langle k_{i}^{\prime}\left|t_{i}^{+}\right| k_{i j}\right\rangle\left\langle k_{i j}\left|t_{j}^{+}\right| k_{j}\right\rangle \\
& \quad+\frac{M(k, Z)\left(\hat{e} \cdot \hat{r}_{j}\right)}{r_{j} r_{i j}^{2}} \frac{m^{3}(2 \pi)^{6}}{\hbar^{6}}\left\langle k\left|t_{j}^{+}\right| k_{i j}^{\prime}\right\rangle\left\langle k_{i j}^{\prime}\left|t_{i}^{+}\right| k_{i j}\right\rangle\left\langle k_{i j}\left|t_{j}^{+}\right| k_{j}\right\rangle \\
& \quad+\frac{M(k, Z)\left(\hat{e} \cdot \hat{r}_{j}\right)}{r_{j} r_{i j}^{2}} \frac{m^{4}(2 \pi)^{8}}{\hbar^{8}}\left\langle k\left|t_{c}^{+}\right| k_{j}^{\prime}\right\rangle\left\langle k_{j}^{\prime}\left|t_{j}^{+}\right| k_{i j}^{\prime}\right\rangle\left\langle k_{i j}^{\prime}\left|t_{i}^{+}\right| k_{i j}\right\rangle\left\langle k_{i j}\left|t_{j}^{+}\right| k_{j}\right\rangle \\
& \text { where } \mathbf{k}_{i j}=k\left(\mathbf{r}_{i}-\mathbf{r}_{j}\right) /\left|\mathbf{r}_{i}-\mathbf{r}_{j}\right|=-\mathbf{k}_{i j}^{\prime}, \\
& \quad \mathbf{k}_{n}=k \hat{r}_{n}=-\mathbf{k}_{n}^{\prime} ; n=i, j
\end{aligned}
$$

and $M(k, Z)$ has been defined in Appendix $\mathrm{A}$.

The matrix elements of $t_{n}^{+}\left(r_{n}\right)$ associated with the atomic potential at $\mathbf{r}=\mathbf{r}_{n}$ may be related to those of $t_{n}^{0}(0)$ as described in Sec. III.

$$
\left\langle k\left|t_{n}^{*}\right| k_{n}\right\rangle=\exp \left[i\left(\mathbf{k}_{n}-\mathbf{k}\right) \cdot \mathbf{r}_{n}\right]\left\langle k\left|t_{n}^{0}\right| k_{n}\right\rangle .
$$

Furthermore, these matrix elements may be expressed in terms of their respective scattering amplitudes:

$$
\left\langle k\left|t_{n}^{0}\right| k_{n}\right\rangle=-\frac{\hbar^{2}}{m(2 \pi)^{2}} f_{n}\left(\theta_{n}\right),
$$

where $\cos \theta_{n}=\hat{k} \cdot \hat{k}_{n}=\hat{k} \cdot \hat{\gamma}_{n}$. Substituting Eqs. (4.6) and (4.7) into Eq. (4.5) and rearranging terms gives: 


$$
\begin{aligned}
& \frac{2 M(k, Z)}{r_{j} r_{i j}}\left(\hat{e} \cdot \hat{r}_{j}\right) \exp \left(-i \mathbf{k} \cdot \mathbf{r}_{i}\right) \exp \left(i k\left|\mathbf{r}_{i}-\mathbf{r}_{j}\right|\right) f_{i}\left(\theta_{k, k_{i j}}\right) \exp \left(i k r_{j}\right) f_{j}(\alpha)+\frac{2 M(k, Z)}{r_{i} r_{j} r_{i j}}\left(\hat{e} \cdot \hat{r}_{j}\right) f_{c}\left(\theta_{k, k_{i}}\right) \\
& \quad \times \exp \left(i k r_{i}\right) \exp \left(i k\left|\mathbf{r}_{i}-\mathbf{r}_{j}\right|\right) f_{i}(\beta) \exp \left(i k r_{j}\right) f_{j}(\alpha)+\frac{M(k, Z)}{r_{j} r_{i j}^{2}}\left(\hat{e} \cdot \hat{r}_{j}\right) \exp \left(-i \mathbf{k} \cdot \mathbf{r}_{j}\right) f_{j}\left(\theta_{k, k_{i j}}\right) \\
& \quad \times \exp \left(2 i k\left|\mathbf{r}_{i}-\mathbf{r}_{j}\right|\right) f_{i}(\pi) \exp \left(i k r_{j}\right) f_{j}(\alpha)+\frac{M(k, Z)}{r_{j}^{2} r_{i j}^{2}}\left(\hat{e} \cdot \hat{r}_{j}\right) f_{c}\left(\theta_{k, k_{j}^{\prime}}\right) \exp \left(2 i k\left|\mathbf{r}_{i}-\mathbf{r}_{j}\right|\right) f_{i}(\pi) \exp \left(2 i k r_{j}\right) f_{j}^{2}(\alpha) .
\end{aligned}
$$

The average $x$-ray absorption cross section for the three-atom system is proportional to the matrix element in Eq. (4.2) averaged over all possible directions $\hat{k}$ of the photoelectron:

$$
\begin{aligned}
\int|\langle f-|\hat{e} \cdot \mathbf{r}| i\rangle|^{2} \frac{d \Omega_{k}}{4 \pi}= & \int \mid M(k, Z)(\hat{e} \cdot \hat{k})+\sum_{n=i, j} \frac{M(k, Z)\left(\hat{e} \cdot \hat{r}_{n}\right)}{r_{n}} f_{n}\left(\theta_{n}\right) \\
& \times \exp \left[2 i k r_{n}\left(1-\cos \theta_{n}\right)\right]+\sum_{n=i, j} \frac{M(k, Z)\left(\hat{e} \cdot \hat{r}_{n}\right)}{r_{n}} f_{n}(\pi) f_{c}\left(\pi-\theta_{n}\right) \exp \left(2 i k r_{n}\right)+\left.(4.8)\right|^{2} \frac{d \Omega_{k}}{4 \pi} .
\end{aligned}
$$

The first three terms in Eq. (4.2) were evaluated in Sec. III, and the results appear in the integrand on the righthand side of Eq. (4.9).

The methods required for evaluation of the angular integrals in Eq. (4.9) have been developed in Appendix B. The results for the lowest order terms in $r$ are:

$$
\begin{aligned}
& \int|M(k, Z)|^{2}(\hat{e} \cdot \hat{k})^{2} \frac{d \Omega_{k}}{4 \pi}=\frac{1}{3}|M(k, Z)|^{2}=\mu_{0}, \\
& 2 \sum_{n=i, j} \frac{|M|^{2}\left(\hat{e} \cdot \hat{r}_{n}\right)}{r_{n}} \int \operatorname{Re}\left\{\exp \left[i k r_{n}\left(1-\cos \theta_{n}\right)\right] f_{n}\left(\theta_{n}\right)(\hat{e} \cdot \hat{k})\right\} \frac{d \Omega_{k}}{4 \pi}=-\sum_{n=i, j} \frac{|M|^{2}\left(\hat{e} \cdot \hat{r}_{n}\right)^{2}}{k r_{n}^{2}} \operatorname{Im}\left[\exp \left(2 i k r_{n}\right) f_{n}(\pi)+f_{n}(0)\right], \\
& 2 \sum_{n=i, j} \frac{|M|^{2}\left(\hat{e} \cdot \hat{r}_{n}\right)}{r_{n}^{2}} \int \operatorname{Re}\left[f_{n}(\pi) f_{c}\left(\pi-\theta_{n}\right) \exp \left(2 i k r_{n}\right)(\hat{e} \cdot \hat{k})\right] \frac{d \Omega_{k}}{4 \pi} \\
& =-\sum_{n=i, j} \frac{|M|^{2}\left(\hat{e} \cdot \hat{r}_{n}\right)^{2}}{k r_{n}^{2}} \operatorname{Im}\left\{\left[\exp \left(2 i \delta_{1}\right)-1\right] f_{n}(\pi) \exp \left(2 i k r_{n}\right\}\right. \\
& \frac{4|M|^{2}\left(\hat{e} \cdot \hat{r}_{j}\right)}{r_{j} r_{i j}} \int \operatorname{Re}\left[\exp \left(i k r_{j}\right) \exp \left(i k\left|\mathbf{r}_{i}-\mathbf{r}_{j}\right|\right) f_{j}(\alpha) \exp \left(-i \hat{k} \cdot \hat{r}_{i}\right)(\hat{e} \cdot \hat{k}) f_{i}\left(\theta_{k, k_{i j}}\right)\right] \frac{d \Omega_{k}}{4 \pi} \\
& =-\frac{2|M|^{2}\left(\hat{e} \cdot \hat{\gamma}_{i}\right)\left(\hat{e} \cdot \hat{r}_{j}\right)}{k r_{i} r_{j} r_{i j}} \operatorname{Im}\left\{\exp \left(i k r_{j}\right) \exp \left(i k\left|\mathbf{r}_{i}-\mathbf{r}_{j}\right|\right) f_{j}(\alpha)\left[\exp \left(i k r_{i}\right) f_{i}(\beta)+\exp \left(-i k r_{i}\right) f_{i}(\pi-\beta)\right]\right\}, \\
& \frac{4|M|^{2}\left(\hat{e} \cdot \hat{r}_{j}\right)}{r_{i} r_{j} r_{i j}} \int \operatorname{Re}\left[\exp \left(i k r_{i}\right) \exp \left(i k r_{j}\right) \exp \left(i k\left|\mathbf{r}_{i}-\mathbf{r}_{j}\right|\right) f_{i}(\beta) f_{j}(\alpha) f_{c}\left(\theta_{k, k_{i}}\right)(\hat{e} \cdot \hat{k})\right] \frac{d \Omega_{k}}{4 \pi} \\
& =-\frac{2|M|^{2}\left(\hat{e} \cdot \hat{r}_{j}\right)\left(\hat{e} \cdot \hat{r}_{j}\right)}{k r_{i} r_{j} r_{i j}} \operatorname{Im}\left\{\exp \left(i k r_{i}\right) \exp \left(i k r_{j}\right) \exp \left(i k\left|\mathbf{r}_{i}-\mathbf{r}_{j}\right|\right) f_{i}(\beta) f_{j}(\alpha)\left[\exp \left(2 i \delta_{1}\right)-1\right]\right\}, \\
& \frac{2|M|^{2}\left(\hat{e} \cdot \hat{r}_{j}\right)}{r_{j} r_{i j}^{2}} \int \operatorname{Re}\left[\exp \left(i k r_{j}\right) \exp \left(2 i k\left|\mathbf{r}_{i}-\mathbf{r}_{j}\right|\right) f_{i}(\pi) f_{j}(\alpha) \exp \left(-i \mathbf{k} \cdot \mathbf{r}_{j}\right)(\hat{e} \cdot \hat{k}) f_{j}\left(\theta_{k, k_{i j}}\right)\right] \frac{d \Omega_{k}}{4 \pi} \\
& =-\frac{|M|^{2}\left(\hat{e} \cdot \hat{r}_{j}\right)^{2}}{k r_{j}^{2} r_{i j}^{2}} \operatorname{Im}\left\{\exp \left(i k r_{j}\right) \exp \left(2 i k\left|\mathbf{r}_{i}-r_{j}\right|\right) f_{i}(\pi) f_{j}(\alpha)\left[\exp \left(i k r_{j}\right) f_{j}(\alpha)+\exp \left(-i k r_{j}\right) f_{j}(\pi-\alpha)\right]\right\}, \\
& \frac{2|M|^{2}\left(\hat{e} \cdot \hat{r}_{j}\right)}{r_{j}^{2} r_{i j}^{2}} \int \operatorname{Re}\left[\exp \left(2 i k r_{j}\right) \exp \left(2 i k\left|r_{i}-r_{j}\right|\right) f_{i}(\pi) f_{j}^{2}(\alpha) f_{c}\left(\theta_{k_{,} k_{j}^{\prime}}\right)(\hat{e} \cdot \hat{k})\right] \frac{d \Omega_{k}}{4 \pi} \\
& =-\frac{|M|^{2}\left(\hat{e} \cdot \hat{r}_{j}\right)^{2}}{k r_{j}^{2} r_{i j}^{2}} \operatorname{Im}\left\{\exp \left(2 i k r_{j}\right) \exp \left(2 i k\left|\mathbf{r}_{i}-\mathbf{r}_{j}\right|\right) f_{i}(\pi) f_{j}^{2}(\alpha)\left[\exp \left(2 i \delta_{i}\right)-1\right]\right\} \text {. }
\end{aligned}
$$

Several cancellations occur in the summation of the terms in Eq. (4.9). The nonoscillatory term containing $f_{n}(0)$ in Eq. (4.11) cancels with the spherically averaged squared term, $\left|f_{n}\left(\theta_{n}\right)\right|^{2}$, by virtue of the optical theorem. The second term in Eq. (4.13) is cancelled by the average of the single scattering cross term arising from atoms $i$ and $j$. The corresponding term in Eq. (4.15) also cancels, but, in this instance, with the averaged cross -terms from the scattering processes shown in diagrams (a) and (e) of Fig. 5.

The expression obtained upon summation of the remaining terms of Eqs. $(4.10)-(4,16)$ is proportional to the absorption coefficient $\mu$ of the system, and may be normalized to $\mu_{0}=\frac{1}{3}|M(k, Z)|^{2}$ to yield the expression for the EXAFS:

$$
\begin{aligned}
\chi(k)= & -\sum_{n=i, j} \frac{3\left(\hat{e} \cdot \hat{r}_{n}\right)^{2}}{k r_{n}^{2}}\left|f_{n}(\pi, k)\right| \sin \left[2 k r_{n}+2 \delta_{i}(k)+\phi_{n}(k)\right]-\frac{6\left(\hat{e} \cdot \hat{r}_{i}\right)\left(\hat{e} \cdot \hat{r}_{j}\right)}{k r_{i} r_{j} r_{i j}}\left|f_{i}(\beta, k)\right|\left|f_{j}(\alpha, k)\right| \sin \left[k\left(r_{i}+r_{j}+r_{i j}\right)+2 \delta_{1}(k)\right. \\
& \left.+\phi_{i}(k)+\phi_{j}(k)\right]-\frac{3\left(\hat{e} \cdot \hat{r}_{j}\right)^{2}}{k r_{j}^{2} r_{i j}^{2}}\left|f_{i}(\pi, k)\right|\left|f_{j}(\alpha, k)\right|^{2} \sin \left[2 k\left(r_{j}+r_{i j}\right)+2 \delta_{1}(k)+\phi_{i}(k)+2 \phi_{j}(k)\right],
\end{aligned}
$$






(a)

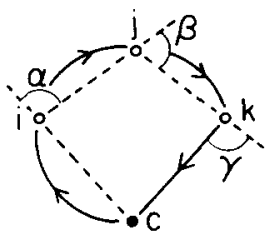

(e)

FIG. 6. (a) -(d) Schematic representation of the terms in the EXAFS expression for a three-atom system [Eq. (4.17)]. (e) Representation of a term in the EXAFS expression for a four-atom system [Eq. (5.1)].

where

$$
f_{n}\left(\theta_{n}\right)=\left|f_{n}\left(\theta_{n}\right)\right| e^{i \Phi_{n}} ; n=i, j \text {. }
$$

Note that $\arg \left[f_{n}\left(\theta_{1}\right)\right]=\arg \left[f_{n}\left(\theta_{2}\right)\right] ; n=i, j$ (i. e. , the phase of the scattering amplitudes is independent of angle for systems comprised of spherically symmetric potentials).

In the case of absorption by polycrystalline samples, an average overall possible polarization directions must be computed, with the results:

$$
\begin{aligned}
& \int\left(\hat{e} \cdot \hat{r}_{n}\right)^{2} \frac{d \Omega_{e}}{4 \pi}=\frac{1}{3}, \\
& \int\left(\hat{e} \cdot \hat{r}_{j}\right)\left(\hat{e} \cdot \hat{r}_{i}\right) \frac{d \Omega_{e}}{4 \pi}=\frac{\hat{r}_{j} \cdot \hat{r}_{i}}{3} .
\end{aligned}
$$

\section{DISCUSSION}

We have presented a straightforward derivation of the basic EXAFS equation. The simplicity of our approach lies in the expansion of the scattering amplitudes of the neighboring atoms about the origin. Accordingly, the phase factor reflecting the difference in path length between unperturbed photoemission and the various scattering processes arises in a natural manner.



FIG. 7. Synthetic EXAFS spectra for Fe-O-Fe system; $\theta=20^{\circ}, r_{j}=r_{i j}=1.76 \AA$. Curves (a) and (b) are the single scattering contributions from the oxygen and iron atoms, respectively. (c) and (d) are the contributions from the multiple scattering terms [the second and third terms in Eq. (4.17)]. (e) is the sum of the above four terms.
The form of the Lippmann-Schwinger equation chosen is of particular significance. $\langle r \mid k\rangle$ represents the outgoing asymptote, and corresponds to the state of the photoelectron after the scattering process. This form has permitted us to describe the scattering in a diagrammatic fashion (Fig. 2). It also emphasizes the interference nature of the EXAFS effect, expressed in Eq. (3.1), in which the probability amplitude for absorption is given by the sum of the amplitudes of three independent scattering processes. Such a sum is required due to the indistinguishability of the individual events: the ejection of a photoelectron in some direction $\hat{k}$ upon ionization is completely indistinguishable from a process whereby the ejected electron scatters off of an adjacent atom and is subsequently scattered in the same direction $\hat{k}$ by the central atom.

Within our formalism, the central atom phase shift cancels in the interference terms since the direct and scattered photoelectron waves are both outgoing in nature. It is thus necessary to include the secondary scattering term in order to retrieve this phase shift. In the alternative form of the Lippmann-Schwinger equation [i.e., the use of $\langle r \mid f+\rangle$ in Eq. (2.5)], however, the term corresponding to the scattered photoelectron is an incoming wave, and no such cancellation occurs. In this latter approach, $\langle r \mid k\rangle$ corresponds to the incoming asymptote of the scattering process, and the phase shift does not occur explicitly within the formalism.

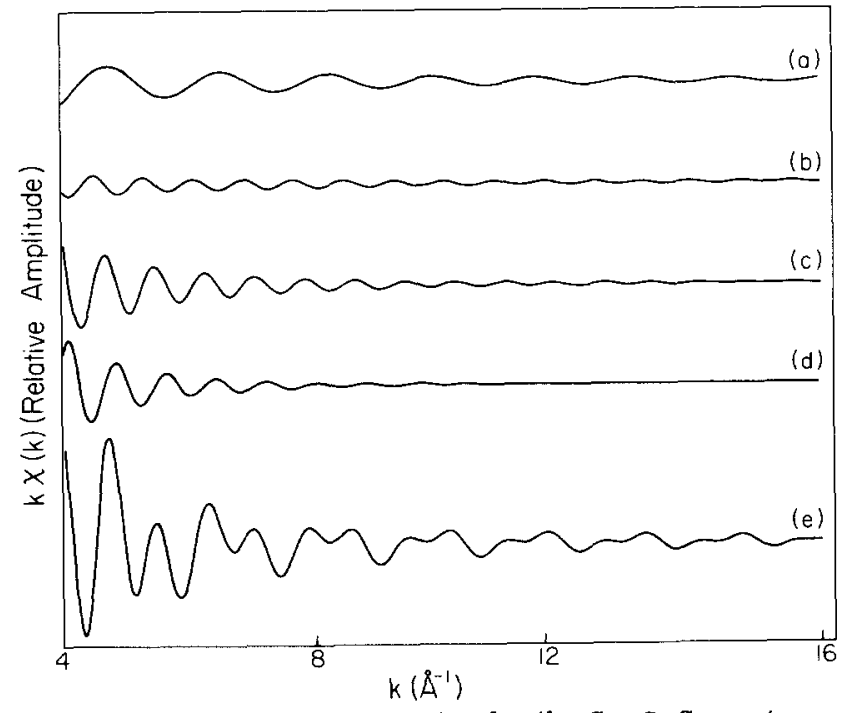

FIG. 8. Synthetic EXAFS spectra for the $\mathrm{Cu}-\mathrm{S}-\mathrm{Cu}$ system; $\theta=20^{\circ}, r_{j}=r_{i j}=2.3 \AA$. The individual components are analogous to those in Fig. 7 . 

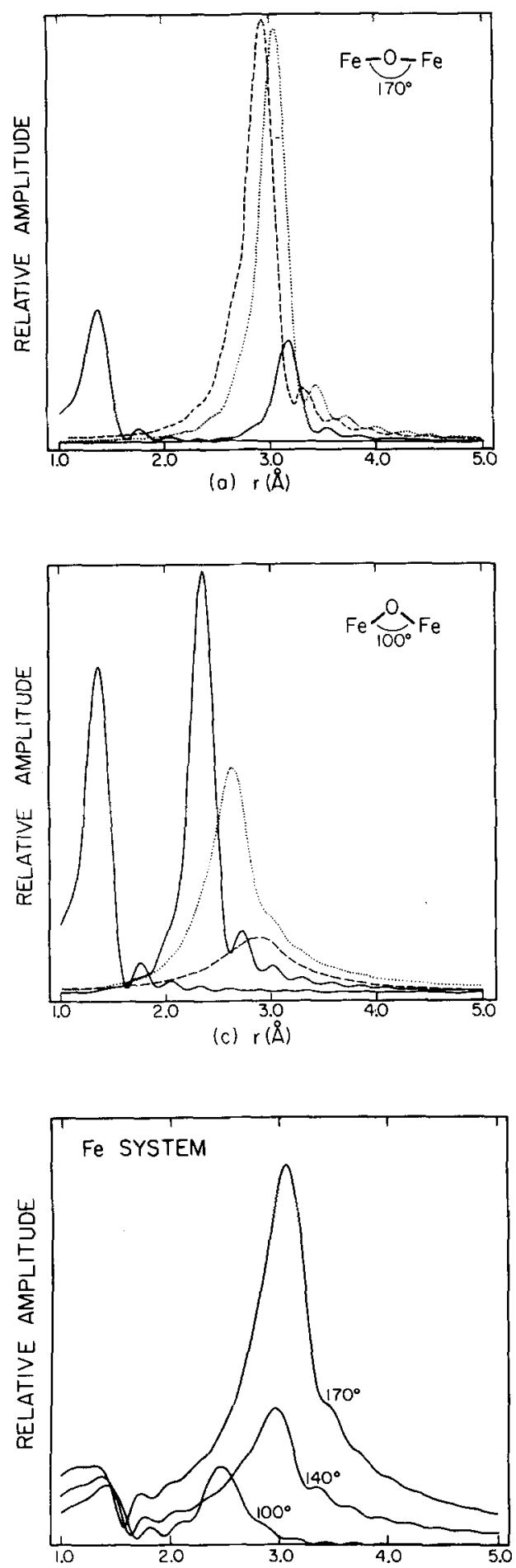

(e) $r(\AA)$



(b) $\operatorname{r}(\stackrel{\AA}{)})$

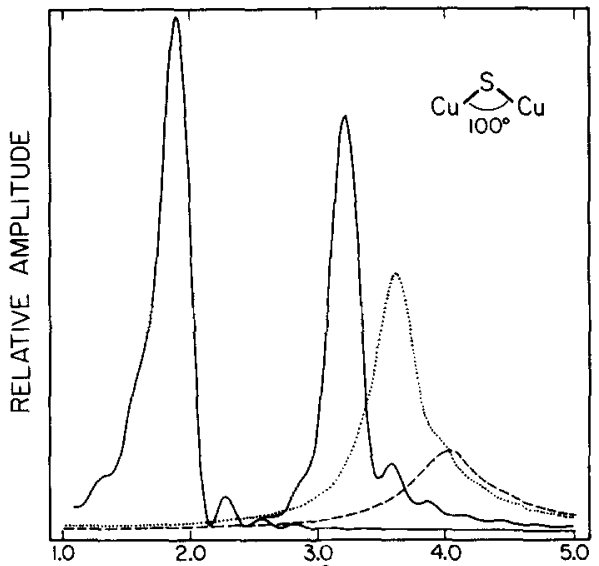

(d) $r(\AA)$

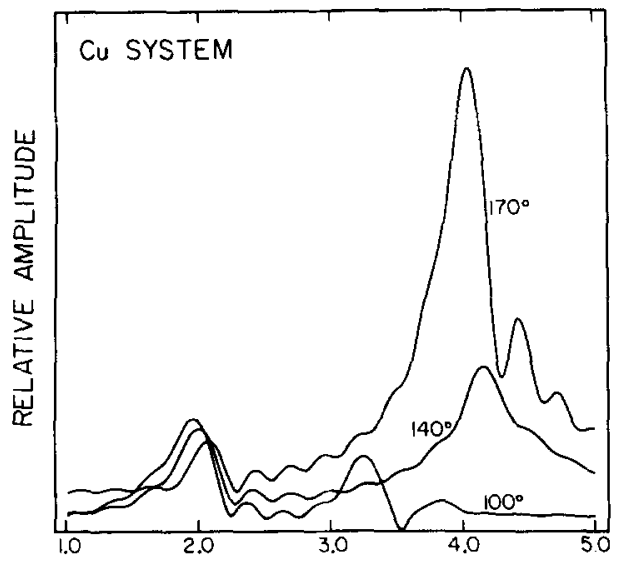

(f) $r(\AA)$

FIG. 9. Representative Fourier transforms of bridged iron and copper systems at different values of the bridging angle. (a)(c) $\mathrm{Fe}-\mathrm{O}-\mathrm{Fe}, r_{j}=r_{i j}=1.76 \AA_{i}$ (d) $\mathrm{Cu}-\mathrm{S}-\mathrm{Cu}, r_{j}=r_{i j}=2.3 \AA$. Solid lines are the single scattering contributions. Dotted and dashed lines are the second and third order multiple scattering contributions, respectively. (e) Fe-O-Fe system; Fourier transform of the sum of the terms in Eq. (4.17). (f) Cu-S-Cu system; Fourier transform of the sum of the terms in Eq. (4.17).

An expression for the oscillatory component of the absorption coefficient for a three-atom system has been given in Eq. (4.17). The first term corresponds to independent single scattering by atoms $i$ and $j$. The second term results from consecutive scattering by atoms $i$ and $j$, and vice versa. Note that this term retains a geometrical dependence even for experiments involving polycry stalline samples [Eq. (4.18)]. The term vanishes when the central angle $\theta$ is $90^{\circ}$; as will occur when atoms $i$ and $j$ both lie in the first shell of a site of octahedral or square planar symmetry. The expression assumed by $\mathrm{Teo}^{28}$ omits this geometrical factor and hence overestimates the contribution of this scattering process to the total EXAFS. The third term in Eq. $(4.17)$ results 

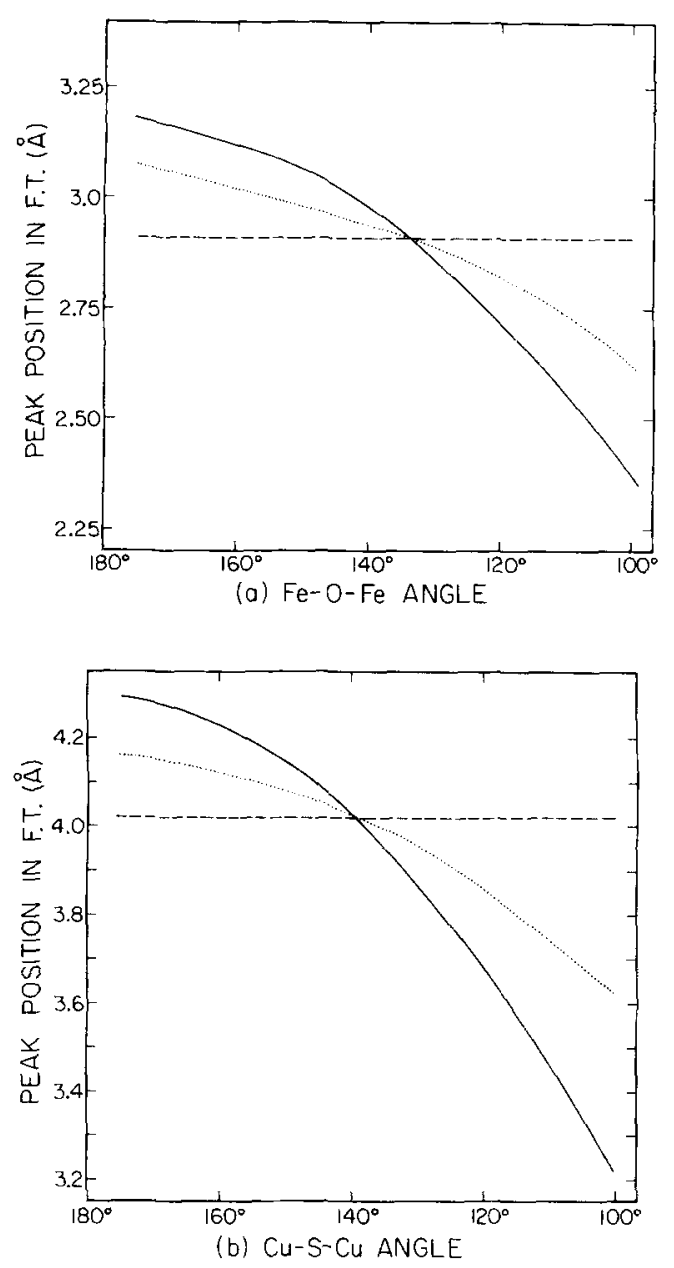

FIG. 10. Peak positions in the Fourier transform as a function of bridging angle for the three scattering processes involving the second-shell atom. The solid lines refer to the second-shell, single scattering term, and the dotted and dashed lines refer to the second and third order multiple scattering terms, respectively.

from a more complex multiple scattering process: the photoelectron scatters sequentially off of atom $j$, atom $i$, and atom $j$ once again (where we have assumed that $\left.\left|r_{j}\right| \leq\left|r_{i}\right|\right)$.

The cancellation of terms that occur in Eq. (4.9) is of particular significance, as it insures that each term in the EXAFS expression is dependent on the sum of the interatomic distances of the process that it represents. These processes are shown diagrammatically in Fig. 6: the first term in Eq. (4.17) corresponds to the sum of diagrams $6(\mathrm{a})$ and $6(\mathrm{~b})$, and the second and third terms correspond to diagrams 6(c) and 6(d), respectively. These diagrams are analogous to those of Fig. 6, but each is the sum of several terms. Note that no scattering is shown by the central atom, and the spherical average of Eq. (4.9) projects out the return path to the origin (shown as a straight line).

Consideration of diagrams like those shown in Fig. 6 permits us to write down by inspection the EXAFS expression resulting from a scattering process of arbitrary complexity. For example, the expression for the fouratom sequential scattering path shown in Fig. 6(e) is

$$
\begin{aligned}
\chi(k)= & -\frac{6\left(\hat{e} \cdot \hat{r}_{j}\right)\left(\hat{e} \cdot \hat{r}_{k}\right)}{k r_{i} r_{i j} r_{j k} r_{k}}\left|f_{i}(\alpha, k)\right|\left|f_{j}(\beta, k)\right|\left|f_{k}(\gamma, k)\right| \\
& \times \sin \left[k\left(r_{i}+r_{i j}+r_{j k}+r_{k}\right)+2 \delta_{1}+\phi_{i}+\phi_{j}+\phi_{k}\right] .(5.1)
\end{aligned}
$$

In order to examine the relative importance of the terms of Eq. (4.17), two-model systems, $\mathrm{Fe}-\mathrm{O}-\mathrm{Fe}$ and $\mathrm{Cu}-\mathrm{S}-\mathrm{Cu}$, have been considered. The respective metal atoms were taken to be the absorbing atoms, and the bond lengths fixed at $1.76 \AA$ for the iron system the distance found in the binuclear complex $\mu$-oxo-bis[tetraphenylporphineiron(III)] and similar compounds $\}$ and $2.3 \AA$ for the $\mathrm{Cu}$ system. The scattering amplitudes were taken from the International Tables for $X-R a y$ Crystallography, Vol. $I V,{ }^{25}$ and the phase shifts from the parametrizations of Lee et al. ${ }^{26}$ The scattering amplitudes used were calculated in the Born approximation. This is an oversimplification, but does not significantly affect the results presented below.

The contribution of each term to the EXAFS of the two systems at a particular value $\left(140^{\circ}\right)$ of the bridging angle are shown in Figs. 7 and 8 . The relative amplitudes of the various terms may be seen in the Fourier transforms of Figs. 9(a) $-9(\mathrm{~d})$. The Fourier transforms of the sum of the four terms contributing to the EXAFS for both systems are shown in Figs. 9(e) and 9(f). Note that the modulus of the sum of the individual terms of Eq. $(4,17)$ is not equal to the sum of their respective moduli. No corrections were made for damping at large $r$ due to inelastic scattering, but any such correction would affect the three terms involving the second-shell atom almost equally (the first-shell peak would be relatively higher, however). Compared with the secondshell, single scattering term, multiple-scattering is most significant in systems in which the ratio of the scattering power of the first-and second-nearest neighbors is large, or when the three atoms are nearly colinear.

Note also the relative linewidths of the various terms. The peaks resulting from multiple scattering processes are broader, primarily because of the dependence on additional scattering amplitudes. Scattering amplitudes, in the Born approximation, have an approximate exponential dependence on $k$, and the effect on the Fourier transform is similar to that of a Debye-Waller factor.

Figures 10 and 11 show the variation of the positions and amplitudes of the peaks in the Fourier transform as a function of bridging angle for the iron and copper systems. Note that, in Figs. 10(a) and 10(b), there is a point where the effect of the additional phase shifts incurred during the multiple scattering processes are exactly offset by the additional path lengths involved in those processes.

The required condition for this crossover point is

$$
\left|\mathbf{r}_{i}\right|-\left|\mathbf{r}_{j}\right|-\left|\mathbf{r}_{i}-\mathbf{r}_{j}\right|=\Delta r_{\phi_{j}},
$$

where $\Delta r_{\phi_{j}}$ is the effective displacement of the peak in the Fourier transform due to the scattering amplitude phase of atom $j . \Delta r_{\phi_{j}}$ is independent of the geometry of the system, and varies over only a limited range for different atoms types $j$. The primary dependence of the crossover point position is on the bond lengths; as the 


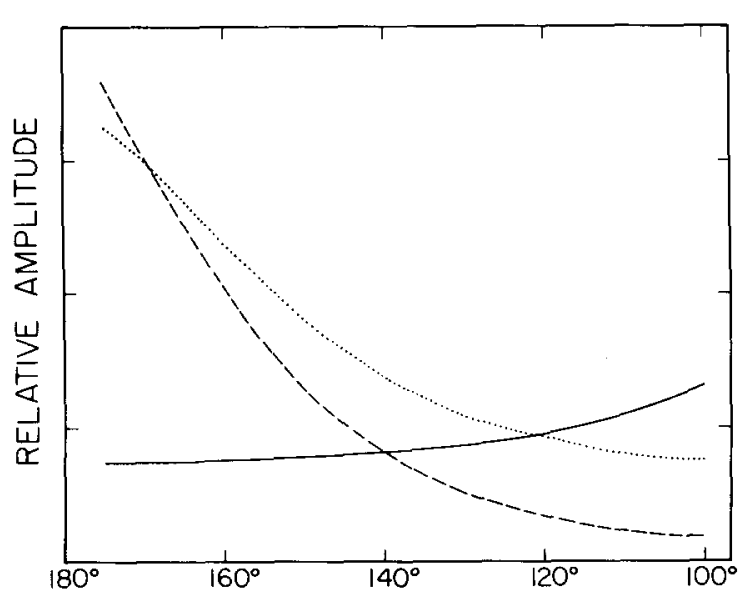

(a) $\mathrm{Fe}-\mathrm{O}-\mathrm{Fe}$ ANGLE

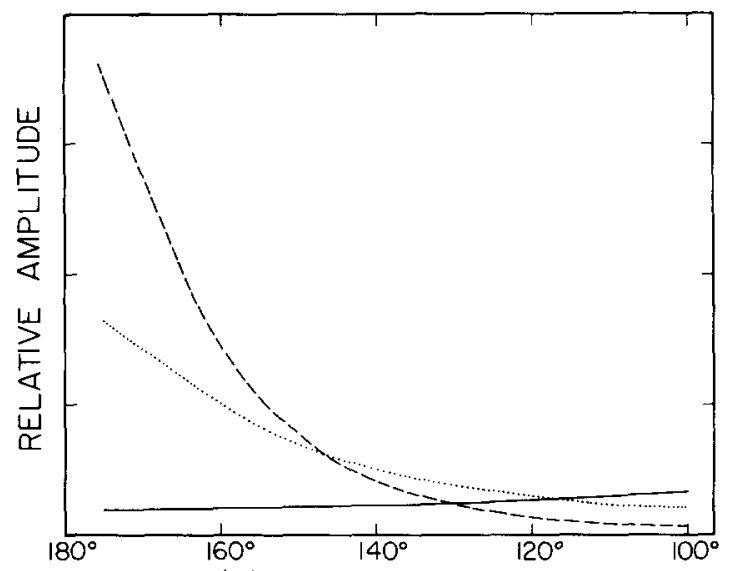

(b) $\mathrm{Cu}-\mathrm{S}-\mathrm{Cu}$ ANGLE

FIG. 11. Relative amplitudes of the peaks in the Fourier transform as a function of bridging angle. The solid lines refer to the second-shell, single scattering term, and the dotted and dashed lines refer to the second and third order multiple scattering terms, respectively. Note that the multiple scattering terms dominate in both systems at bridging angles exceeding about $120^{\circ}$.

distances in the system increase, the crossover point occurs at larger values of the bridging angle.

At bridging angles greater than that of the crossover point, the multiple scattering terms are of particular importance. As shown in Fig. 11 these terms are of large amplitude at high angles and, above the crossover point, the corresponding peaks in the Fourier transform occur at smaller values of $r$ than does the second-shell, single scattering peak. In general, the three peaks involving scattering by the second-shell atom will be close enough to overlap. The presence of the multiple scattering terms will then cause an apparent increase in amplitude of the second-nearest neighbor peak, and will move it to a smaller value of $r$.

At bridging angles smaller than that of the crossover point, the compound second-shell peak will appear at larger values of $r$ than would the single scattering peak; but the effect is less significant because the multiplescattering components are smaller in this region. Multiple scattering effects have recently been observed by $\mathrm{Co}$ et al. ${ }^{27}$ and are in qualitative agreement with the theoretical predictions presented above.

In summary, multiple..scattering effects need to be considered in most problems in which non-nearest neighbor shells are of interest. This is often the case in investigations of active sites in metalloproteins and similar compounds. However, careful analysis of these effects will permit the elucidation of geometrical information which is not otherwise available from the single scattering theory.

\section{ACKNOWLEDGMENTS}

The authors wish to thank Dr. Ronald C. Gamble for encouragement and numerous helpful discussions. This is contribution no. 6448 from the Arthur Amos Noyes Laboratory of Chemical Physics.

\section{APPENDIX A}

We wish to evaluate the integral

$$
I=\int\langle k \mid r\rangle \hat{e} \cdot \mathbf{r}\langle r \mid i\rangle d \mathbf{r}
$$

which appears in Eq. (3.1); where $\langle r \mid k\rangle=(2 \pi)^{-3 / 2}$ $\times \exp (i \mathbf{k} \cdot \mathbf{r})$ and $\langle r \mid i\rangle=(\pi)^{-1 / 2}\left(Z / a_{0}\right)^{3 / 2} \exp \left(-Z r / a_{0}\right)$.

Expanding Eq. (A1) in terms of spherical harmonics, $I$ may be rewritten as

$$
\begin{aligned}
I= & (2 \pi)^{-3 / 2} 4(\pi)^{1 / 2}\left(\frac{Z}{a_{0}}\right)^{3 / 2} \sum_{l} \sum_{m} \int(-i)^{l} j_{l}(k r) \\
& \times \exp \left(-Z r / a_{0}\right) r^{2} d r \int Y_{l}^{* m}\left(\Omega_{k}\right) Y_{l}\left(\Omega_{r}\right)(\mathbf{r} \cdot \hat{e}) d \Omega_{r},
\end{aligned}
$$

where $d \mathbf{r}=r^{2} d r d \Omega_{r}$.

The angular integration in Eq. (A2) may be performed using the additional theorem for spherical harmonics:

$$
\begin{aligned}
& \sum_{l} \sum_{m} \int Y_{l}^{* m}\left(\Omega_{k}\right) Y_{l}^{m}\left(\Omega_{r}\right)(\mathbf{r} \cdot \hat{e}) d \Omega_{r} \\
& \quad=\frac{4 \pi r}{3} \sum_{l}^{\infty} \sum_{m=-l}^{l} \sum_{g=-1}^{1} Y_{1}^{\xi}\left(\Omega_{e}\right) Y_{l}^{* m}\left(\Omega_{k}\right) \int Y_{1}^{*}\left(\Omega_{r}\right) Y_{l}^{m}\left(\Omega_{r}\right) d \Omega_{r} \\
& =\frac{4 \pi r}{3} \sum_{g=-1}^{1} Y_{1}^{g}\left(\Omega_{e}\right) Y_{l}^{* m}\left(\Omega_{k}\right) \delta_{m, e} \delta_{l, 1}=r(\hat{k} \cdot \hat{e})
\end{aligned}
$$

Substituting Eq. (A3) into Eq. (A2), the expression for. I becomes

$$
\begin{aligned}
I= & (2 \pi)^{-3 / 2} 4(\pi)^{1 / 2}(-i)\left(\frac{Z}{a_{0}}\right)^{3 / 2}(\hat{k} \cdot \hat{e}) \\
& \times \int_{0}^{\infty} j_{1}(k r) \exp \left(-Z r / a_{0}\right) r^{3} d r,
\end{aligned}
$$

where $j_{1}(k r)=(k r)^{-2} \sin k r-(k r)^{-1} \cos k r$. Making use of the definite integral,

$$
\int_{0}^{\infty} x^{n} e^{-\mu x} d x=n ! \mu^{-(n+1)}, \operatorname{Re} \mu>0
$$

the radial integration in Eq. (A4) may be performed to obtain

$$
I=M(k, Z)(\hat{k} \cdot \hat{e}),
$$

where $M(k, Z)=-i\left[(2)^{1 / 2} / \pi\right]\left[8 k\left(Z / a_{0}\right)^{5 / 2} /\left(Z^{2} / a_{0}^{2}+k^{2}\right)^{3}\right]$. 


\section{APPENDIX B}

In this appendix, the angular integrals in Eq. (3.16), (3.17), and (3.19) are evaluated.

The first of these corresponds to $\mu_{0}$, the hypothetical absorption coefficient in the absence of neighboring atoms. Placing $\hat{e}$ along the $z$ direction:

$$
\begin{aligned}
\mu_{0} & \propto \frac{1}{4 \pi} \int|M|^{2} \hat{k} \cdot \hat{e} d \Omega_{k} \\
& =\frac{|M|^{2}}{4 \pi} \int \cos ^{2} \theta_{k} \sin \theta_{k} d \theta_{k} d \phi_{k}=\frac{1}{3}|M(k, Z)|^{2} .
\end{aligned}
$$

The following result is required in Eq. (3.17):

$$
I=\int(\hat{e} \cdot \hat{k}) \exp \left(-i \mathbf{k} \cdot \mathbf{r}_{j}\right) f\left(\theta_{j}\right) \frac{d \Omega_{k}}{4 \pi}
$$

$(\hat{e} \cdot \hat{k})$ may be expanded in spherical harmonics, and setting $r_{j}$ along the $z$ axis, the azimuthal integration yields the $m=0$ component in the expansion. Hence:

$I=-\left(\hat{e} \cdot \hat{r}_{j}\right) \frac{1}{2} \int d\left(\cos \theta_{k}\right) \cos \theta_{k} \exp \left(-i \mathbf{k} \cdot \mathbf{r}_{j}\right) f\left(\theta_{j}\right)$,

where $\cos \theta_{k}=\left(\hat{k} \cdot \hat{r}_{j}\right)$. exp $\left(-i \mathbf{k} \cdot \mathbf{r}_{j}\right)$ and $f\left(\theta_{j}\right)$ may be expressed in terms of Legendre polynomials:

$$
\begin{aligned}
& \exp \left(-i \mathbf{k} \circ \mathbf{r}_{j}\right)=\sum_{l}(2 l+1)(-i)^{l} j_{l}\left(k r_{j}\right) P_{l}\left(\cos \theta_{k}\right), \\
& f\left(\theta_{j}\right)=\sum_{l^{\prime}} f_{l^{\prime}} P_{l^{\prime}}\left(\cos \theta_{k}\right),
\end{aligned}
$$

and

$$
P_{l}\left(\cos \theta_{k}\right)=\left(\frac{4 \pi}{2 l+1}\right)^{1 / 2} Y_{l}^{0}\left(\Omega_{k}\right)
$$

Substituting the above into $\mathrm{Eq}$. (B1) yields

$$
\begin{aligned}
I= & \sum_{l, l^{\prime}}\left(\hat{e} \cdot \hat{r}_{j}\right) \frac{1}{2}(2 l+1)(-i)^{l}\left(\frac{4 \pi}{2 l+1}\right)^{1 / 2}\left(\frac{4 \pi}{2 l^{\prime}+1}\right)^{1 / 2}\left(\frac{4 \pi}{3}\right)^{1 / 2} \\
& \times j_{l}\left(k r_{j}\right) f_{l^{\prime}} \int Y_{l^{\prime}}^{0}\left(\Omega_{k}\right) Y_{1}^{0}\left(\Omega_{k}\right) Y_{l}^{0}\left(\Omega_{k}\right) d\left(-\cos \theta_{k}\right),
\end{aligned}
$$

where $d \Omega_{k}=d\left(-\cos \theta_{k}\right) d \phi_{k}$, and $\int_{0}^{2 r}\left(d \phi_{k} / 2 \pi\right)=1$. The angular integral in Eq. (B5) may be evaluated using the properties of Clebsch-Gordan coefficients ${ }^{24}$ :

$\int Y_{l^{*}}^{0}\left(\Omega_{k}\right) Y_{1}^{0}\left(\Omega_{k}\right) Y_{l}^{0}\left(\Omega_{k}\right) d \Omega_{k}$

$$
=\left[\frac{\left(2 l^{\prime}+1\right)}{4 \pi} \frac{3}{(2 l+1)}\right]^{1 / 2}\left[C\left(l^{\prime}, 1, l ; 0,0,0\right)\right]^{2},
$$

where $\left|l-l^{\prime}\right| \leq 1 \leq\left|l+l^{\prime}\right|$ and $l+l^{\prime}+1=2 n$ ( $n$ an integer). The summation over $l$ and $l^{\prime}$ may be replaced by a single sum that has two components $l \pm 1$. Using the explicit forms of the Clebsch-Gordan coefficients above,

$$
\begin{aligned}
I= & \sum_{l}\left(\hat{e} \cdot \hat{r}_{j}\right) f_{l}\left[(-i)^{l+1}\left(\frac{l+1}{2 l+1}\right) j_{l+1}\left(k r_{j}\right)\right. \\
& \left.+(-1)^{l-1}\left(\frac{l}{2 l+1}\right) j_{l-1}\left(k r_{j}\right)\right]=\sum_{l}\left(\hat{e} \cdot \hat{r}_{j}\right) f_{l}\left[\frac{l}{2 l+1} j_{l-1}\left(k r_{j}\right)\right. \\
& \left.-\left(\frac{l+1}{2 l+1}\right) j_{l+1}\left(k r_{j}\right)\right](-i)^{l-1}
\end{aligned}
$$

Using the asymptotic form of the spherical Bessel function:

$$
\begin{aligned}
& j_{l}\left(k r_{j}\right) \frac{1}{k r_{j}+\infty} \frac{1}{k r_{j}} \sin \left(k r_{j}-\frac{1}{2} l \pi\right) \\
& \quad=\frac{1}{k r_{j}}\left[\sin \left(k r_{j}\right) \cos \left(\frac{1}{2} l \pi\right)-\cos \left(k r_{j}\right) \sin \left(\frac{1}{2} l \pi\right)\right] .
\end{aligned}
$$

Equation (B6) may be expanded in $\exp \left( \pm i k r_{f}\right) . P_{3}(\cos 0)$ $=(1)^{l}$ and $P_{l}(\cos \pi)=(-1)^{l}$; and the final form of $I$ is

$$
I=\left(\hat{e} \cdot \hat{r}_{j}\right) \frac{1}{k r_{j}} \frac{1}{2} i\left[\exp \left(i k r_{j}\right) f(\pi)+\exp \left(-i k r_{j}\right) f(0)\right]
$$

Hence:

$$
\begin{aligned}
\int 2 \operatorname{Re} & {\left[M^{*}(\hat{k} \cdot \hat{e})(3.12)\right] \frac{d \Omega_{k}}{4 \pi} } \\
& =-\sum_{j}|M|^{2} \frac{1}{k r_{j}^{2}}\left(\hat{e} \cdot \hat{r}_{j}\right)^{2} \operatorname{Im}\left[\exp \left(2 i k r_{j}\right) f(\pi)+f(0)\right] .
\end{aligned}
$$

The third angular integration necessary is given in Eq. (3.19):

$$
I^{\prime}=\int(\hat{e} \cdot \hat{k}) f_{c}\left(\pi-\theta_{j}\right) \frac{d \Omega_{k}}{4 \pi},
$$

where $f_{c}\left(\pi-\theta_{j}\right)=(-1)^{l} f_{c}\left(\theta_{j}\right)$

$$
=\sum_{l} \sum_{m=-l}^{l} \frac{(-1)^{l}}{2 i k}(4 \pi)\left[\exp \left(2 i \delta_{l}\right)-1\right] Y_{l}^{m}\left(\Omega_{k}\right) Y_{l}^{* m}\left(\Omega r_{j}\right) .
$$

Expanding $(\hat{e} \cdot \hat{k})$ in spherical harmonics:

$$
(\hat{e} \cdot \hat{k})=\frac{4 \pi}{3} \sum_{m^{*}=-1}^{1} Y_{1}^{m^{\prime}}\left(\Omega_{e}\right) Y_{1}^{* m^{*}}\left(\Omega_{k}\right) .
$$

Equation (B7) may be written:

$$
\begin{aligned}
I^{\prime}= & \frac{(-1)^{l}}{2 i k} \frac{4 \pi}{3} \sum_{l} \sum_{m=-l}^{l} \sum_{m^{\prime}=-1}^{1}\left[\exp \left(2 i \delta_{l}\right)-1\right] Y_{l}^{*}\left(\Omega r_{j}\right) Y_{1}^{m^{\prime}}\left(\Omega_{e}\right) \\
& \times \int Y_{l}^{m}\left(\Omega_{k}\right) Y_{1}^{* m^{\prime}}\left(\Omega_{k}\right) d \Omega_{k}=-\frac{1}{2 i k}\left[\exp \left(2 i \delta_{1}\right)-1\right]\left(\hat{e} \cdot \hat{r}_{j}\right)
\end{aligned}
$$

due to the orthogonality of spherical harmonics. Hence:

$$
\begin{aligned}
\int 2 \operatorname{Re}\left[M^{*}(k, Z)(\hat{e} \cdot \hat{k})(3.13)\right] \frac{d \Omega_{k}}{4 \pi} \\
=-|M|^{2} \sum_{j} \frac{\left(\hat{e} \cdot \hat{r}_{j}\right)^{2}}{k r_{j}^{2}} \operatorname{Im}\left\{\left[\exp \left(2 i \delta_{1}\right)-1\right] f_{j}(\pi) \exp \left(2 i k r_{j}\right)\right\},
\end{aligned}
$$

${ }^{1}$ R. de L. Kronig, Z. Phys. 70, 317 (1931); 75, 191 (1932); 75,468 (1932).

${ }^{2}$ H. Petersen, Z. Phys, 80, 258 (1933).

${ }^{3}$ A. I. Kosterev, Zh. Eksp. Teor. Fiz. 19, 413 (1949).

${ }^{4}$ T. Shiraiwa, T. Ishimura, and M. Sawada, J. Phys. Soc. Jpn. 138, 848 (1958).

${ }^{5}$ D. E. Sayers, F. W. Lytle, and E. A. Stern, Adv. X-ray Anal. 13, 248 (1970).

${ }^{6}$ W. L. Schiach, Phys. Rev. B 8, 4028 (1973).

${ }^{7}$ E. A. Stern, Phys. Rev. B 10, 3027 (1974).

${ }^{8}$ C. A. Ashley and S. Doniach, Phys, Rev. B 11, 1279 (1975).

${ }^{9}$ P. A. Lee and T. B. Pendry, Phys. Rev, B 11, 2795 (1975).

${ }^{10}$ P. A. Lee, Phys. Rev. B 13, 5261 (1976).

${ }^{11}$ P. A. Lee and G. Beni, Phys. Rev, B 15, 2862 (1977).

${ }^{12}$ P. A. Lee, P. H. Citrin, P. Elsenberger, and B. M. Kincaid, Rev. Mod. Phys. 53, 769 (1981).

${ }^{13}$ E. A. Stern, Contemp. Phys. 18, 289 (1978).

${ }^{14}$ L. V. Azaroff, Rev. Mod. Phys, 35, 1012 (1963).

${ }^{15}$ V. V. Schmidt, Bull. Acad. Sci. USSR, Ser. Phys. 25, 988 (1961).

${ }^{16} \mathrm{E}$. Merzbacher, Quantum Mechanics, 2nd ed. (Wiley, New 
York, 1970), p. 466.

${ }^{17} \mathrm{~J}$. R. Taylor, Scattering Theory (Wiley, New York, 1972), p. 168. Equation (2.4) is a second order inhomogeneous differential equation, and its solution is given by the sum of the solution to the homogeneous equation (i.e., with $V=0$ ) together with any solution of the inhomogeneous equation.

${ }^{18}$ P. Lloyd and P. V. Smith, Adv. Phys. 21, 69 (1972).

${ }^{19}$ L. I. Schiff, Quantum Mechanics, 3rd ed. (McGraw-Hill, New York, 1968), p. 338.

${ }^{20}$ M. Lax, Rev. Mod. Phys. 23, 287 (1951).

${ }^{21}$ J. R. Taylor, Ref. 19, p. 43.

${ }^{22}$ Reference 19, p. 54.
${ }^{23}$ B. K. Teo, P. A. Lee, A. L. Simons, P. Eisenberger, and B. M. Kincaid, J. Am. Chem. Soc. 99, 3854 (1977).

${ }^{24}$ E. Merzbacher, Ref. 19 , p. 396.

${ }^{25} \mathrm{~J}$. A. Ibers and W. C. Hamilton, International Tables for $X$-ray Crystallography (Kynoch, Birmingham, U. K., 1974), Vol, IV, p. 152.

${ }^{26}$ P. A. Lee, B. K. Teo, and A. L. Simons, J. Am. Chem. Soc. 99, 3856 (1977).

${ }^{27}$ M. S. Co, K. O. Hodgson, W. A. Hendrickson, and J. L. Smith, Synchrotron Radiation Laboratory Report 81/02 Proposal No. 388 , VII-37.

${ }^{28}$ B. K. Teo, J. Am. Chem. Soc. 103, 3990 (1981). 